\title{
Treatment of unstable osteochondritis dissecans in adults with autogenous osteochondral grafts (Mosaicplasty): long-term results
}

\author{
MARIO RONGA ${ }^{1}$, PLACIDO STISSI ${ }^{1}$, GIUSEPPE LA BARBERA ${ }^{1}$, MARCO VALOROSO $^{1}$, \\ GLORIA ANGERETTI², EUGENIO GENOVESE ${ }^{3}$, PAOLO CHERUBINO ${ }^{1}$
}

${ }^{1}$ Orthopaedics and Traumatology, Department of Biotechnology and Life Sciences (DBSV), University of Insubria, Varese, Italy ${ }^{2}$ Radiology Unit, Department of Surgical and Morphological Sciences, University of Insubria, Varese, Italy

${ }^{3}$ Department of Radiology, University of Cagliari, Italy

\begin{abstract}
Purpose: the unstable osteochondritis dissecans (OCD-type II and III according to the ICRS classification) of the knee largher than $>2.5 \mathrm{~cm}^{2}$ in adults are uncommon lesions and there is no consensus on how to treat them. Medium-term studies have reported good results using autogenous osteochondral plugs (mosaicplasty). The aim of this study is to analyze the long-term results of this technique for the treatment of unstable OCD in a selected group of adult patients.

Methods: four patients with OCD at either one of the femoral condyles were included in this prospective study. The average age was 21.2 years (range, 18-24 years). The OCD lesions were classified as type II in three patients and type III in one patient and the average size was $3.8 \mathrm{~cm}^{2}$ (range, 2.55-5.1 $\mathrm{cm}^{2}$ ). The lesions were treated in situ with a variable number of autogenous osteochondral plugs ( Modified Cincinnati, Lysholm II and Tegner scores were used for clinical and functional evaluation. Magnetic resonance arthrography (MRA) was performed before surgery and at 2, 5 and 10 years after surgery. A modified MOCART score was used to evaluate MRA findings.
\end{abstract}

\section{Corresponding Author:}

Mario Ronga, MD

Orthopaedics and Traumatology, Department of Biotechnology

and Life Sciences (DBSV)

University of Insubria, Ospedale di Circolo

Viale L. Borri 57, 21100 Varese, Italy

E-mail: mario.ronga@uninsubria.it
Results: the average follow-up duration was ten years and 6 months (range, 10-11 years). No complications occurred. At the final follow-up, all scores (clinical, functional and MOCART) improved. In all but one of the patients MRA showed complete osteochondral repair.

Conclusions: the fixation of large and unstable OCD lesions with mosaicplasty may be a good option for treating type II or III OCD lesions in adults. The advantages of this technique include stable fixation, promotion of blood supply to the base of the OCD fragment, and grafting of autologous cancellous bone that stimulates healing with preservation of the articular surface.

Level of evidence: Level IV, therapeutic case series.

Keywords: osteochondritis dissecans, osteochondral graft, adults, MR arthrography.

\section{Introduction}

Osteochondritis dissecans (OCD) is a common lesion that mainly affects children and adolescents with a prevalence of approximately 15-29 cases per 100,000 (1). The exact prevalence in adults is unknown. Large $\left(>2.5 \mathrm{~cm}^{2}\right)$ unstable lesions, classified as type II (partial discontinuity, stable on probing) or type III (complete discontinuity, "dead in situ", not dislocated osteochondral fragment) by the International Cartilage Repair Society (ICRS) (2), are rare lesions in adults that require surgical treatment due to their limited healing potential and more deleterious clinical course compared with juvenile OCD (3). Several techniques 
to address these lesions have been described (drilling and fixation with metal or absorbable screws, pins and bone pegs), with success rates of 80 to $90 \%$ of patients $(1,4)$. However, most previous studies are limited by, small case series that do not allow separate analysis of results in juvenile and adult patients, the different indications considered, the absence of comparison between different fixation methods, and their only short- or medium-term follow-up. Moreover, this type of surgery is not devoid of complications related to metal screw removal (when needed), biodegradation of synthetic polymers and host response $(5,6)$. The aims of surgical treatment in OCD should include maintenance of joint congruity, rigid fixation of unstable fragments, and biological repair of the osteochondral fragment $(7,8)$. In 1999, Berlet et al. (9) described a new technique designed to fix unstable OCD fragments and to stimulate the healing response; this technique used autologous osteochondral plugs in the same manner as in the original mosaicplasty technique. Two studies are reported in literature with good or excellent results ranging between 95 and $91 \%$ at medium-term follow-up $(10,11)$.

In this prospective cohort study, a selected group of adult patients undergoing fixation of unstable OCD (ICRS type II or III) of the knee with autologous osteochondral plugs (mosaicplasty) was evaluated clinically, functionally and with magnetic resonance arthrography (MRA). The study hypothesis was that this technique is an effective treatment for these lesions.

\section{Methods}

Study design

The present study was designed as a prospective cohort study with a minimum follow-up of 10 years after the index surgery.

\section{Patients}

All patients gave written informed consent before entering the study. Inclusion criteria were: adult patients with symptomatic, unstable OCD lesions (ICRS type II or III) of the femoral condyles larger than $2.5 \mathrm{~cm}^{2}$ at MRA evaluation, non-responsive to conservative treatment for more than 6 months, and who practiced sports at least at recreational level. Patients were excluded from the present study if one or more of the following findings were present: metabolic diseases, a history of patellofemoral pa thology, meniscal or ligamentous tear requiring repair or reconstruction, and previous surgery of the affected knee.

The patients underwent clinical examination and the following imaging studies: anteroposterior, standing weight-bearing, and lateral radiographs of the injured knee, and skyline views of both patellae with the knees at $30^{\circ}$ of flexion, and MRA. Knee examination under anesthesia and diagnostic arthroscopy were performed in each patient at the time of the index surgery. OCD lesion type, were classified according to the ICRS rating system (2). Macroscopic assessment was then compared with MRA findings.

\section{Surgical technique and rehabilitation protocol}

A diagnostic arthroscopy using standard anteromedial and anterolateral portals was performed with the patient supine and a tourniquet applied to the thigh. The OCD lesion was assessed for position, size (using an arthroscopic rod), and stability (using a probe). The lesions were treated in situ with a variable number of osteochondral plugs $\left(\varnothing 4.5 \mathrm{~mm}^{2}\right)$ harvested from the edges of the trochlea using the MOSAICPLASTYTM System (Smith \& Nephew, Inc., Andover, MA - USA) as described by Berlet et al. (9). Briefly, osteochondral plugs were inserted until rigid stability of the OCD lesion was obtained starting from the center of the lesion. The plugs length was calculated, on preoperative MRI aiming to pass at least as far into the normal underlying subchondral bone as the distance it has passed through the lesion and across the interface (Fig. 1). Donor sites were filled with fibrin glue (Tissucol, Baxter Spa, Deerfield, Illinois, USA). After wound closure, the joint was covered with a compressive elastic bandage.

Postoperatively, isometric strengthening of the quadriceps and unrestricted range of motion began on the first day, while partial weight bearing was allowed after 6 weeks and full weight bearing shortly thereafter. The timing of return to sports depended on the type of joint impact involved in the specific activity: sports involving low loading stresses on the knee (e.g. swimming and cycling) could be resumed three months after surgery, while return to activities involving jumping, twisting and running (e.g. soccer and basketball) was not allowed for at least six months. 

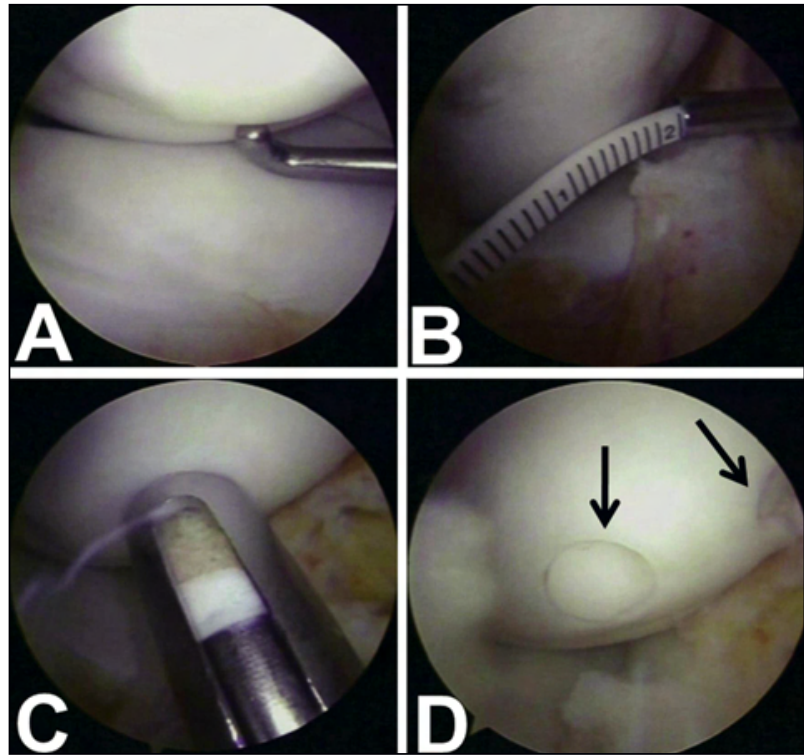

Fig. 1. Case 2. A. Arthroscopic view of type II OCD lesion of the medial femoral condyle. B. The OCD fragment measures $3 \times 1.7 \mathrm{~cm}$. C. The osteochondral plug is inserted in the recipient site with the plunger. D. Final view with two plugs that match the surrounding articular surface (arrows).

\section{Outcome evaluations}

Clinical and functional evaluations were performed by an independent observer (PS), blinded to the surgical treatment, using the modified Cincinnati rating system (12), the Lysholm function score (13), and the Tegner activity scale (14) at regular intervals: preoperatively and at 2, 4, 8, 12, and 24 weeks after surgery, and then annually. Patients were also asked whether they had returned to their sport and, if so, whether they had returned to the same, a lower or a higher level compared to the preinjury level.

MRA was performed preoperatively, as well as at 2, 5 and 10 years postoperatively using a dedicated coil (quad knee) with a 1.5-T tomograph: Eclipse (PickerMarconi, Koninklijke Philips, Netherlands) (unit 1) and Avanto (Siemens, Erlangen, Germany) (unit 2). All patients gave their written informed consent before the procedure. MRA was performed after intra-articular injection of $40 \mathrm{ml}$ paramagnetic contrast agent (Magnevist $2 \mathrm{mmol} / \mathrm{l}$; Bayer-Schering, Berlin, Ger many; Dotarem $2.5 \mathrm{mmol} / \mathrm{l}$; Guerbet, Villepinte, France). The "unit 1" MRA protocol involved the use of sagittal, coronal or axial SE T1 sequences; sagittal or coronal fat-saturated FSE PD/T2 sequences; and 3D fat-saturated FSE T1 sequences in axial or coronal pla- nes. The "unit 2" protocol consisted of 3D isotropic T1 sequences in the axial plane; sagittal and coronal TSE T1 sequences; and sagittal or coronal fat-saturated FSE PD/T2 sequences. An independent observer (GA), blinded to the surgical treatment, evaluated the MRA findings using the modified MOCART scoring scale (15).

\section{Data analysis}

Statistical comparison between preoperative and postoperative values was not possible, owing to the limited data.

\section{Results}

The study included 4 patients ( 3 males and 1 female) with a mean age of 21.2 years (range, 18-24 years) at the time of surgery. The mean follow-up was 10 years and 6 months (range, 10 - 11 years). The OCD lesions were classified as ICRS type II in 3 patients and type III in 1 patient with an average size of $3.8 \mathrm{~cm}^{2}$ (range, $2.55-5.1 \mathrm{~cm}^{2}$ ) at preoperative MRA. In all cases the arthroscopic evaluation confirmed the lesion type and size. In three cases the medial femoral condyle was involved and in one case the lateral femoral condyle. Two osteochondral plugs were used in two cases and three osteochondral plugs in the other two cases (Tab. 1). No complications were observed postoperatively or at long-term follow-up. At the time of the latest follow-up the mean modified Cincinnati rating system score increased from $3.75 \pm 1.25$ preoperatively to $9.25 \pm 0.95$, the mean Lysholm score from $44.5 \pm 5.32$ to $96.5 \pm 4.35$, the mean Tegner scale score from $3 \pm$ 1.15 to $6 \pm 1.41$, and the mean modified MOCART scale score from $40 \pm 4.08$ to $66.25 \pm 8.53$ (Figs. 2, 3). In 1 case the modified MOCART score was less than 60 points (Fig. 4). At an average follow-up of 6.5 months (range, 5-8 months), all patients returned to sports at the same preinjury level.

\section{Discussion}

Juvenile OCD lesions are typically stable and have the potential to heal with non-surgical treatment whereas adult OCD lesions are more likely to show instability (1). The gold-standard surgical treatment for large 
Table 1. Patient data.

\begin{tabular}{llllllll}
\hline Case no. & Age & Sex & Site & Size $\mathbf{( \mathbf { c m } ^ { 2 } )}$ & ICRS classification & $\begin{array}{l}\text { No. of } \\
\text { osteochondral } \\
\text { plugs }\end{array}$ \\
\hline 1 & 21 & M & L-LFC & 3.00 & II & 2 & 132 \\
(mollow-up \\
2 & 18 & M & R-MFC & 5.10 & II & 3 & 126 \\
3 & 22 & F & L-MFC & 2.55 & II & 2 & 126 \\
4 & 24 & M & L-MFC & 4.55 & III & 3 & 120 \\
\hline
\end{tabular}

${ }^{a}$ ICRS, International Cartilage Repair Society; M, male; F, female; L, left; R, right; LFC, lateral femoral condyle; MFC, medial femoral condyle.

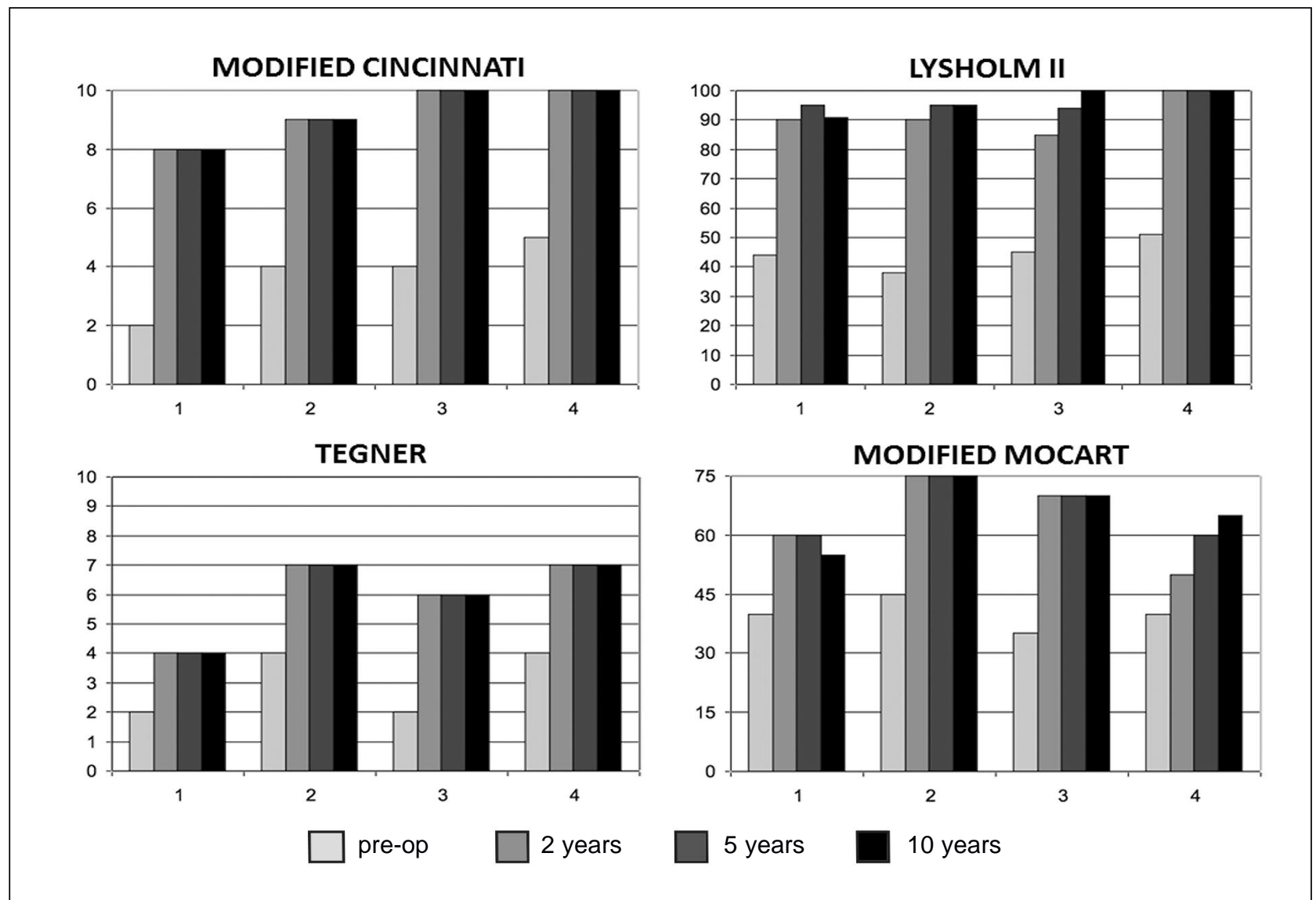

Fig. 2. Histograms showing the preoperative and postoperative scores on various clinical and functional evaluation scales of 4 patients at preop, 2,5 and 10 years follow-up.

unstable OCD lesions in adults is still debated. Different techniques such as drilling and fixation with metal screws or simple pins and biodegradable implants have been proposed with not predictable results due to a not homogenous and small case series, different indications (juvenile and adult OCD) and fixation methods (3, 4, 8, 16-19) considered, the results of these techniques cannot be considered predictable. Various complications have been reported, such as cartilage lesions, bone necrosis, bone loosening following metal screw removal, synovitis and implant breaking up with the formation of loose 


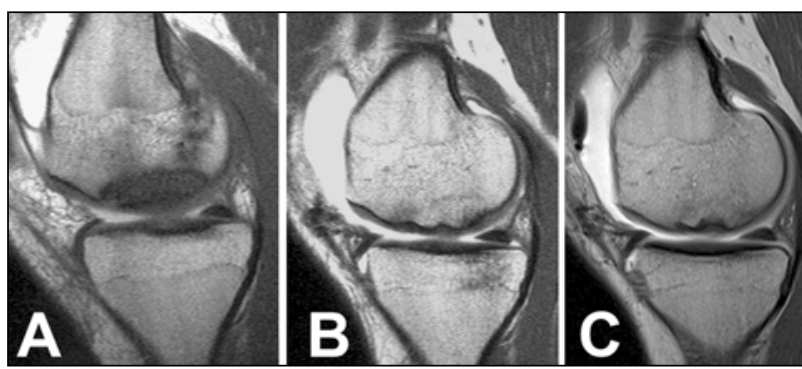

Fig. 3. Case 2. MR arthrography (Sagittal oblique SE T1-weighted imaging). A. Type II OCD lesion of the medial femoral condyle with sclerotic interface between the OCD fragment and the healthy bone. B-C. rotic interface between the OCD fragment and the healthy bone. B-C.
2 and 10 years after surgery. Repair of the OCD fragment that shows 2 and 10 years after surgery. Repair of the OCD fragment that shows bone. Disappearance of the sclerotic interface with continuous articular cartilage surface.

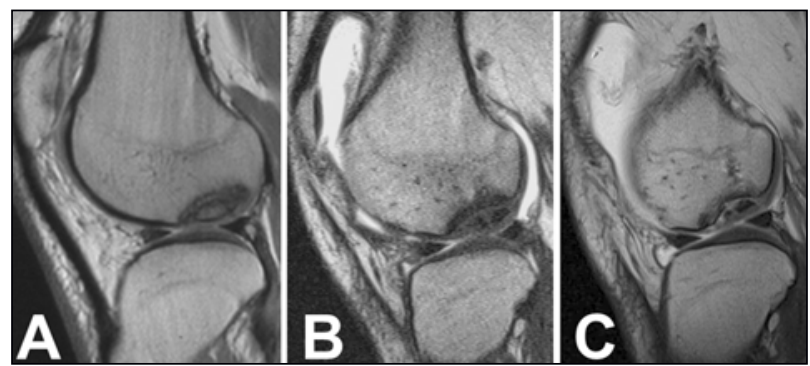

Fig. 4. Case 1. MR arthrography (Sagittal oblique SE T1-weighted imaging). A. Type II OCD lesion of the medial femoral condyle. B-C. 2 and 10 years after surgery. A progressive bony fragmentation of the OCD can be appreciated despite the continuous articular cartilage surface. No bone marrow edema is evident and there is a partial interruption of the sclerotic interface.

bodies when biodegradable implants were used (4-6). If failure occurs, fragmentation or loss of the bony component of the OCD fragment creates many problems in reconstructing the osteochondral unit and reproducing the normal condyle shape (7). Moreover, many of these methods stabilize the fragment in the absence of biological healing of the OCD fragment and between the fragment and the surrounding tissue (1). The goals of surgical treatment should be to promote healing through breaking of the sclerotic interface between the OCD fragment and the healthy bone, increasing of the blood supply to the base of the fragment by drilling, bone grafting with autologous cells and growth factors across the fragment and its base, and to obtain stable fixation leaving the OCD fragment as intact as possible $(1,7,9)$. In 1999, Berlet et al. (9) proposed a modified mosaicplasty technique to fix in situ unstable OCD with autologous osteochondral plugs. Two studies with an average follow-up of 3.4 and 4.5 years reported good or excellent results in 95 and $91 \%$ of patients respectively $(10,11)$. Our study confirms the efficacy of this technique, with good results obtained at long-term follow-up and all patients returning to sport at the same level as prior to their injury. We used 4.5-mm-diameter osteochondral plugs because a previous study demonstrated that grafts with a diameter of $3.5 \mathrm{~mm}$ or less did not have sufficient biomechanical strength and would collapse, whereas $6.5-\mathrm{mm}$-diameter grafts led to degenerative changes at the patellofemoral joint (20). The number of plugs used depends on the lesion size: two plugs for lesions smaller than $3 \mathrm{~cm}^{2}$, three plugs for lesions larger than $3 \mathrm{~cm}^{2}$ (11). We did not observe any complication related to the osteochondral plug harvesting. We filled the donor sites with fibrin glue to reduce bleeding and to stimulate fibrocartilaginous repair (21). Several Authors have reported no donor-site morbidity problems when four osteochondral grafts of $5.5-\mathrm{mm}$-diameter harvested from the margins of the femoral trochlea were used $(22,23)$. In all but one of our patients MRA showed complete osteochondral repair with disappearance of the sclerotic interface between the OCD fragment and the healthy bone and a signal indicating revascularization of the fragment. MRA did not reveal any irregularities of the articular surface and showed good integration of the cartilage of the OCD fragment with the surrounding tissue. We used MRA because it can show the presence of minimal clefts between the OCD fragment and the condyle and between the osteochondral plugs and the surrounding cartilage, otherwise no evident at standard MR (15). In all cases the arthroscopic evaluation confirmed the type and size of the lesions highlighted by MRA. A discrepancy between good clinical results and an insufficient MOCART score was found in just one patient. We suppose that the partial interruption of the sclerotic interface, the absence of bone marrow edema and the integrity of articular cartilage could explain the clinical outcome, despite the MR appearance.

Major strengths of the present study are its prospective nature and the long-term follow-up. The patient selection process was very accurate, so that the procedure was performed only in patients without knee malalignment, meniscal or ligamentous lesions. Furthermore, two independent observers blinded to the surgical treatment performed clinical and MRI evaluations. Weaknesses of this study are the relatively small number of patients included and the lack of a 
control group. However, large type II-III OCD lesions in a selected group of adult patients without any concomitant articular lesions are not common, and it would have been next to impossible to collect a number of patients large enough to randomize into two groups for a single orthopaedic department. Previously, Miniaci and Tytherleigh-Strong (10) reported a series of 20 patients that included only 9 adults, while the 12 patients reported by Miura et al. (11) included only 3 adults. Moreover, previous or concomitant surgeries were reported in 8 out of 20 and 2 out of 12 patients respectively (11). These findings can be considered a bias when evaluating the efficacy of the procedure. The prognosis for healing of an OCD lesion after surgery depends on skeletal maturity, and in younger patients with open physes the prognosis is more favorable (1). Previous or concomitant surgery can jeopardize the final result of a cartilage repair procedure (4).

On the basis of the good clinical and MRA results obtained at long-term follow-up, mosaicplasty can be considered a safe and reliable option for the treatment of unstable OCD in adult patients. The biological and mechanical principles of this procedure allow to treat these complex lesions in a simple way preserving the OCD fragments. The superiority of this technique over others can only be established through an adequately multicentre powered randomized controlled trial with long term follow-up.

\section{References}

1. Kocher MS, Tucker R, Ganley TJ, et al. Management of osteochondritis dissecans of the knee: current concepts review. Am J Sports Med. 2006;34:1181-1191.

2. International Cartilage Repair Society. ICRS Cartilage Injury Evaluation Package. The International Cartilage Repair Society website: http://www.cartilage.org/Evaluation_ Package/ICRS_Evaluation.pdf. Accessed 6 March 2002.

3. Lindén B. The incidence of osteochondritis dissecans in the condyles of the femur. Acta Orthop Scand. 1976;47:664-667.

4. Sgaglione NA, Miniaci A, Gillogly SD, et al. Update on advanced surgical techniques in the treatment of traumatic focal articular cartilage lesions in the knee. Arthroscopy. 2002;18 (2 Suppl 1): 9-32.

5. Friederichs MG, Greis PE, Burks RT. Pitfalls associated with fixation of osteochondritis dissecans fragments using bioabsorbable screws. Arthroscopy. 2001;17:542-545.

6. Kim SJ, Shin SJ. Loose bodies after arthroscopic osteochondral autograft in osteochondritis dissecans of the knee. Arthroscopy. 2000;16: E16.

7. Madry H, van Dijk CN, Mueller-Gerbl M. The basic science of the subchondral bone. Knee Surg Sports Traumatol Arthrosc. 2010;18:419-33.

8. Smillie IS. Treatment of osteochondritis dissecans. J Bone Joint Surg Br. 1957;39-B:248-260.

9. Berlet GC, Mascia A, Miniaci A. Treatment of unstable osteochondritis dissecans lesions of the knee using autogenous osteochondral grafts (mosaicplasty). Arthroscopy. 1999;15:312-316.

10. Miniaci A, Tytherleigh-Strong G. Fixation of unstable osteochondritis dissecans lesions of the knee using arthroscopic autogenous osteochondral grafting (mosaicplasty). Arthroscopy. 2007;23:845-851.

11. Miura K, Ishibashi Y, Tsuda E, et al. Results of arthroscopic fixation of osteochondritis dissecans lesion of the knee with cylindrical autogenous osteochondral plugs. Am J Sports Med. 2007;35:216-22.

12. Noyes FR, McGinniss GH, Mooar LA. Functional disability in the anterior cruciate insufficient knee syndrome. Review of knee rating systems and projected risk factors in determining treatment. Sports Med. 1984;1:278-302.

13. Lysholm J, Gillquist J. Evaluation of knee ligament surgery results with special emphasis on use of a scoring scale. Am J Sports Med. 1982;10:150-154.

14. Tegner Y, Lysholm J. Rating systems in the evaluation of knee ligament injuries. Clin Orthop Relat Res. 1985;(198):43-49.

15. Genovese E, Ronga M, Angeretti MG, et al. Matrix-induced autologous chondrocyte implantation of the knee: mid-term and long-term follow-up by MR arthrography. Skeletal Radiol. 2011;40:47-56.

16. Johnson LL, Uitvlugt G, Austin MD, et al. Osteochondritis dissecans of the knee: arthroscopic compression screw fixation. Arthroscopy. 1990; 6:179-189.

17. Slough JA, Noto AM, Schmidt TL. Tibial cortical bone peg fixation in osteochondritis dissecans of the knee. Clin Orthop Relat Res. 1991;(267):122-127.

18. Thomson NL. Osteochondritis dissecans and osteochondral fragments managed by Herbert compression screw fixation. Clin Orthop Relat Res. 1987;(224):71-78.

19. Victoroff BN, Marcus RE, Deutsch A. Arthroscopic bone peg fixation in the treatment of osteochondritis dissecans in the knee. Arthroscopy. 1996;12:506-509.

20. Evans PJ, Miniaci A, Hurtig MB. Manual punch versus power harvesting of osteochondral grafts. Arthroscopy. 2004;20: 306-310.

21. Schlag G, Redl H. Fibrin sealant in orthopedic surgery. Clin Orthop Relat Res. 1988;227:269-285.

22. Gudas R, Kalesinskas RJ, Kimtys V, et al. A prospective randomized clinical study of mosaic osteochondral autologous transplantation versus microfracture for the treatment of osteochondral defects in the knee joint in young athletes. Arthroscopy. 2005;21:1066-1075.

23. Hangody L, Vásárhelyi G, Hangody LR, et al. Autologous osteochondral grafting-technique and long-term results. Injury. 2008;39 Suppl 1:S32-S39. 